\title{
Strategies leading to drug incorporation by the public health system: the Brazilian Society of Clinical Oncology experience
}

\author{
Estratégias para incorporação de novas drogas pelo sistema publico de saúde: \\ a experiencia da Sociedade Brasileira de Oncologia Clínica \\ Gustavo dos Santos Fernandes¹, Cinthya Sternberg²
}

Brazil is the largest country in Latin America and is the ninth largest economy by nominal GDP in the world and the eighth largest by purchasing power parity. Brazilian health care system is complex and harbors severe inequity issues leading to disparities in access to novel expensive therapeutic agents, especially in the public health system that, despite their scientifically confirmed clinical benefits, are not available to the vast majority of the population.

Keeping the balance between efficacy, cost and ethical availability of state of the art drugs is a main problem in a country that has a long history of inequity and faces one of the worst financial crisis ever. The incorporation of novel therapies must be assessed regarding its positive clinical benefit on the public health care patients and the financial burden that its prescription would impose to the public health budget. This is a multifaceted discussion, and several stakeholders are involved in the process - from health authorities to pharmaceutical companies, researchers, and civil society. ${ }^{(1)}$

At the beginning of our term at the Board of Directors of the Brazilian Society of Clinical Oncology (SBOC/2015-2017), we have tried to find means to assist the Brazilian Public Health System (Sistema Único de Saúde - SUS) to select drugs regarded, according to scientific evidence available, as beneficial therapies that should be accessible to our population. The process started by compiling and comparing all the available therapeutic agents in Brazil, both in public and private practice (the ones licensed by the Brazilian regulatory health agency - ANVISA). We have found 37 indications there were available on private but not on the public health system, and the resulting list was presented to the Federal Council of Medicine and to de Ministry of Health. As the list was extensive, it was very unlikely that the budget of the Ministry of Health would cover all the listed indications and make them available to the population in general. Therefore, we undertook the task of meeting with experts and the Board of Directors of SBOC to choose the drugs that should be submitted to the appreciation of CONITEC (National Commission of Incorporation of New Technologies at SUS), and hopefully, subsequently made available to the public. The first drug brought

1. Brazilian Society of Clinical Oncology, Hospital Sirio-Libanês, São Paulo, SP, Brazil.

2. Brazilian Society of Clinical Oncology, Graduate Program of Anatomical Pathology, Faculty of Medicine, Universidade Federal do Rio de Janeiro, Rio de Janeiro, RJ, Brazil.

\footnotetext{
Conflicts of interest: no

Corresponding author: Cinthya Sternberg

Phone: +55 (21) 994480150. E-mail: sternbergcinthya@gmail.com

Received on: September 10, 2017 | Accepted on: September 28, 2017

DOI:10.26790/BJO20171345CE
} 
forward was anti-her2 (trastuzumab + pertuzumab) therapy for metastatic Her-2 positive breast cancer. In order add real life information to the submission file, we have performed a retrospective analysis were we estimated that not offering those drugs to selected patients would represent more than 30,000 years of life lost because of lack of access by the brazilian population. 2 Subsequently, we performed a cost effectiveness analysis and involved the pharmaceutical company that produces the aforementioned drugs on the process and largely discussed with them the extent of discount could be negotiated by the government to render the technology feasible to be incorporated onto the public practice. Finally, we have submitted our dossier to CONITEC, also involving patient advocacy organizations on the process. Recently, trastuzumab was approved as monotherapy directed to Her2-positive metastatic breast cancer patients for use in the public health system, which encompasses more than 150 million people and should benefit approximately 1,408 women in 2018 if they receive chemotherapy plus trastuzumab. If our original petition was approved, there would be 1,576 women alive in 2018 if they receive the gold standard of chemotherapy plus trastuzumab and pertuzumab. ${ }^{(2)}$

Historically, medical Societies in Brazil abstained from getting involved in the process of pressing the government for the incorporation of novel therapies, maintaining a censor position on industry, government and insurance companies, but rarely assuming the protagonist position on those initiatives. On reporting our experience, we would like to stress that ethical cooperation between specialists, government, patients and pharmaceutical industries can lead to improvements in health care access even in a country facing an economic crisis such as Brazil. We are reproducing the same process for two other indications and expect to achieve the same results and, ultimately, to improve patient care and clinical benefits.

\section{REFERENCES}

1. Ades F. Access to oncology drugs in Brazil: juggling innovation and sustainability in developing countries. Med Access @ point care 2017; 1(1): e10 - e15.

2. Debiasi M, Reinert T, Kaliks R, Amorim G, Caleffi M, Sampaio

C, Fernandes GDS, Barrios CH. Estimation of Premature Deaths From Lack of Access to Anti-HER2 Therapy for Advanced Breast Cancer in the Brazilian Public Health System. J Glob Oncol. 2016;3(3):201-7. 